\title{
NSAIDs protect neurons and preserve memory in a mouse model of AD
}

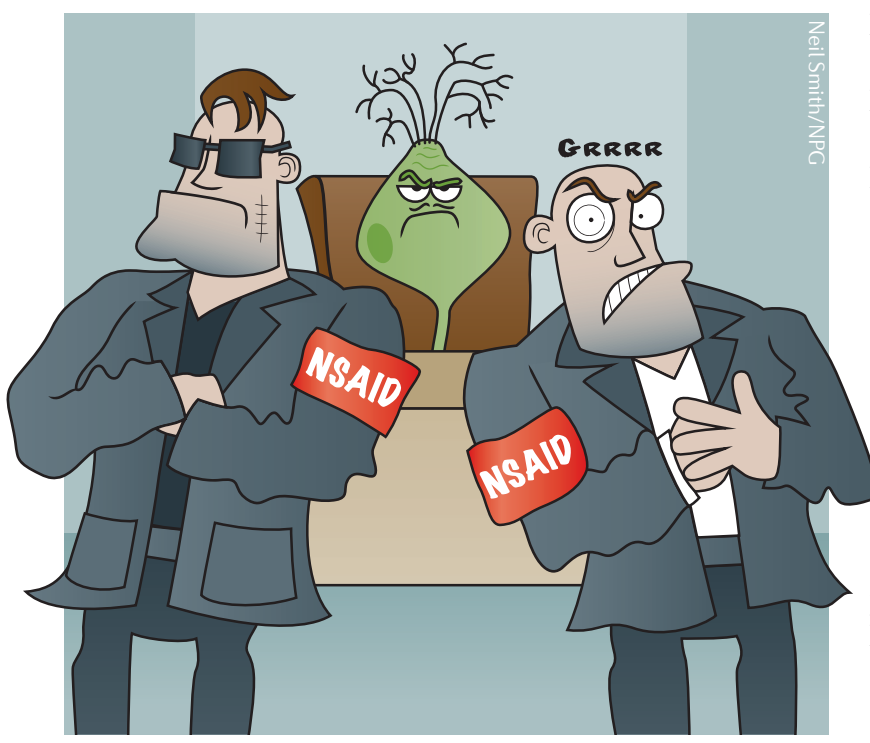

$4 c$

Ibuprofen

... protected

the mice

from object

recognition

memory

impairment
Ibuprofen might protect neurons from damage associated with amyloid- $\beta_{42}$ $\left(\mathrm{A} \beta_{42}\right)$ accumulation, according to a new study in Alzheimer disease (AD) model mice. Moreover, ibuprofen treatment prevented the development of early memory impairment.

NSAIDs inhibit cyclooxygenases (COX-1 and COX-2), which are known for their role in promoting inflammation via prostaglandin synthesis. Epidemiological studies have linked NSAID use with reduced risk of $\mathrm{AD}$, but the mechanisms underlying the association have remained elusive. Some mouse model studies have reported that the protective role of NSAIDs is attributable to their anti-inflammatory function, whereas others have observed the effect to be independent of inflammation.

Katrin Andreasson and colleagues from Stanford University, California, USA, administered ibuprofen to APP-PS1 mice. APPPS1 mice - an established animal model of AD - develop AD-like pathology, including $A \beta$ accumulation, and subsequent memory impairment. "Ibuprofen treatment did not alleviate neuroinflammation or reduce $A \beta$ deposition, however, the treatment protected the mice from impairment of object recognition memory, which is an early manifestation of $\mathrm{AD}$ pathology in APP-PS1 mice," Andreasson comments.

Previous studies have shown that $\mathrm{A} \beta$ oligomers induce glial COX-2 expression. Using cultured mouse hippocampal cells, Andreasson and co-workers discovered that $\mathrm{A} \beta_{42}$ also induces COX-2 expression in neurons, and that ibuprofen blocked this effect. According to the authors, neuronal COX-2 activity could, therefore, be the target for NSAID protective action early in the disease, whereas glial COX activity could be more relevant to later stages of disease.

Moreover, the researchers found that ibuprofen altered the expression of genes related to synaptic plasticity. Among the regulated genes was Tdo2, which encodes an enzyme that synthesizes kynurenine. Aberrant activation of the kynurenine pathway has been linked to neurodegeneration by previous research, and Andreasson and her team discovered that neuronal Tdo2 expression in APP-PS1 mice contributed to the observed novel object recognition memory deficit.

Given that long-term use of NSAIDs is associated with a risk of adverse effects, neuroprotection with NSAIDs is unlikely to be safe in humans. However, the findings could help understand the pathophysiological mechanisms of AD. "Modulation of the kynurenine pathway could be a factor associated with maintenance of normal memory function," Andreasson concludes.

Hemi Malkki

ORIGINAL ARTICLE Woodling, N. S. et al. Cyclooxygenase inhibition targets neurons to prevent early behavioural decline in Alzheimer's disease model mice. Brain http://dx.doi. org/10.1093/brain/aww117 (2016) 